Recibido: 12/07/2019 --- Aceptado: 19/02/2019 --- Publicado: 15/07/2020

\title{
USO DE REDES SOCIALES DIGITALES COMO ESTRATEGIA DE MERCADOTECNIA EN PYMES PIROTÉCNICAS DE TULTEPEC EN EL ESTADO DE MÉXICO
}

\section{USE OF DIGITAL SOCIAL MEDIA AS A MARKETING STRATEGY IN PYROTECHNIC SMES OF TULTEPEC IN THE STATE OF MEXICO}

8II Alan Roberto Villagómez Manrique ${ }^{1}$. Instituto Politécnico Nacional (IPN). México. phys.villa@outlook.com

iD 83) Elizabeth Acosta Gonzaga². Instituto Politécnico Nacional (IPN). México. elz.acosta.gonzaga@gmail.com

\section{RESUMEN}

El uso de redes sociales digitales (RSD) como estrategia de mercadotecnia se ha convertido en una práctica fundamental para las empresas de todo el mundo. En México, el $99.8 \%$ de las empresas son de tamaño pequeño y mediano (pymes) y, pese a su importancia, existe poca literatura sobre el uso de RSD como estrategia de mercadotecnia en las pymes mexicanas. Asimismo, la industria pirotécnica de Tultepec, Edo. de Méx., produce el $50 \%$ de la pirotécnica nacional, emplea hasta el $60 \%$ de la población local y se compone principalmente por pymes. Dada la relevancia de este sistema productivo, el objetivo de la investigación es determinar el grado de adopción de RSD como estrategia de mercadotecnia por parte de las empresas pirotécnicas de Tultepec, los principales usos que les dan y, en su ausencia, las razones para no utilizarlas. Para ello, se aplicaron cuestionarios a los dueños de diversas empresas del encadenamiento productivo de pirotecnia (tiendas de materias primas, fabricantes e intermediarios) y se encontró que sólo el $15 \%$ de los encuestados utilizan RSD como estrategia de mercadotecnia y las utilizan, principalmente para el posicionamiento de la marca y alcanzar mayores audiencias. Finalmente, se encontró que la principal razón para no utilizarlas es la inseguridad y experiencias relacionadas con la delincuencia. Los resultados obtenidos, exhiben la necesidad de una estrategia de capacitación para los empresarios que conduzca a un uso seguro y eficaz de RSD como herramienta de mercadotecnia.

\footnotetext{
${ }^{1}$ Alan Roberto Villagómez Manrique: Estudiante de la Maestría en Ciencias en Estudios Interdisciplinarios para pymes en la Unidad Profesional Interdisciplinaria de Ingeniería y Ciencias Sociales y Administrativas (UPIICSA) del Instituto Politécnico Nacional.

phys.villa@outlook.com

2Elizabeth Acosta Gonzaga: Profesora-Investigadora de la Sección de Estudios de Posgrado e Investigación de la Unidad Profesional Interdisciplinaria de Ingeniería y Ciencias Sociales y Administrativas (UPIICSA) del Instituto Politécnico Nacional.

eacostag@ipn.mx
} 
Villagómez Manrique, A. R. y Acosta Gonzaga, E. Uso de redes sociales digitales como

estrategia de mercadotecnia en pymes pirotécnicas de Tultepec en el estado de México

PALABRAS ClAVE: redes sociales digitales - usos - mercadotecnia - México pirotecnia - pymes - inseguridad.

\section{ABSTRACT}

The use of digital social media (DSM) as a marketing strategy has become a fundamental practice for companies around the world. In Mexico, $99.8 \%$ of enterprises are small and medium-sized (SMEs) and, despite their importance, there is little literature on the use of DSM as a marketing strategy in Mexican SMEs. Also, the pyrotechnic industry of Tultepec, State of Mexico, produces $50 \%$ of the national pyrotechnics, employs up to $60 \%$ of the local population and is composed mainly of SMEs. Given the relevance of this production system, the objective of this piece of research is to determine the percentage of Tultepec pyrotechnics companies that use DSM as a marketing strategy, the main uses they are given and, in their absence, the reasons for not using them. To this end, questionnaires were applied to the owners of various companies in the pyrotechnics production chain (raw material stores, manufacturers and intermediaries) and it was found that only $15 \%$ of respondents use DSM as a marketing strategy and use them mainly to position the brand and reach larger audiences. Finally, it was found that the main reason for not using them is the insecurity and experiences related to crime. The results obtained show the need for a training strategy for business owners that leads to a safe and effective use of DSM as a marketing tool.

KEYWORDS: digital social media - uses - marketing - Mexico - pyrotechnics smes - insecurity.

\section{USO DAS REDES SOCIAIS DIGITAIS COMO ESTRATÉGIA DE MARKETING EM PME PIROTÉCNICAS DE TULTEPEC NO ESTADO DE MÉXICO}

\section{RESUMO}

O uso das redes sociais digitais (RSD) como estratégia de marketing se converteu numa prática fundamental para as empresas do mundo inteiro. No México, o 99.8\% das empresas são de pequeno e médio porte (PME) e, apesar da sua importância, existe pouca literatura sobre o uso de RSD como estratégia de marketing nas PME mexicanas. Da mesma forma, a indústria pirotécnica de Tultepec, Edo. do Méx., produz $50 \%$ da pirotecnia nacional, emprega até $60 \%$ da população local e se compõe principalmente por PME. Dada à relevância deste sistema produtivo, 0 objetivo da pesquisa é determinar o grau de acolhimento das RSD como estratégia de marketing por parte das empresas pirotécnicas de Tultepec, os principais usos que eles lhes dão e, na sua falta, os motivos para não usa-as. Para isto, se aplicaram questionários aos donos de diversas empresas da cadeia produtiva de pirotecnia (lojas de matérias-primas, fabricantes e intermediários) e se descobriu que somente $15 \%$ dos entrevistados usam RSD como estratégia de marketing e as utilizam, 
Villagómez Manrique, A. R. y Acosta Gonzaga, E. Uso de redes sociales digitales como

estrategia de mercadotecnia en pymes pirotécnicas de Tultepec en el estado de México

principalmente para o posicionamento da marca e para conseguir maiores audiências. Finalmente, se encontrou que a principal motivação para não utilizá-las é a insegurança e experiências relacionadas com a delinquência. Os resultados obtidos, mostram a necessidade de uma estratégia de capacitação para os empresários que leve ao uso seguro e eficaz das RSD como ferramenta de marketing.

PALAVRAS CHAVE: redes sociais digitais - usos - marketing - México - pirotecnia - PME - insegurança.

\section{Cómo citar el artículo:}

Villagómez Manrique, A. R. y Acosta Gonzaga, E. (2020). Uso de redes sociales digitales como estrategia de mercadotecnia en pymes pirotécnicas de Tultepec en el estado de México. [Use of digital social media as a marketing strategy in pyrotechnic smes of Tultepec in the state of Mexico]. Revista de Comunicación de la SEECI, (52), 73-93. doi: http://doi.org/10.15198/seeci.2020.52.73-93

Recuperado de http://www.seeci.net/revista/index.php/seeci/article/view/610

\section{INTRODUCCIÓN}

En México, las micro, pequeñas y medianas empresas (pymes) tienen un papel preponderante dentro de la economía. De acuerdo con los datos de los Censos Económicos realizados por el Instituto Nacional de Estadística y Geografía (INEGI), en el 2014 se registraron 5.654.014 unidades económicas, de las cuáles 99,8\% tienen menos de 251 empleados; es decir, más del 99\% de las unidades económicas en México pertenecen a la categoría pymes. Además, se encontró que las pymes generan 7 de cada 10 empleos y contribuyeron con el $35,9 \%$ de producto interno bruto (PIB) nacional en el 2013; siendo el comercio la actividad económica que genera mayor número de empleos.

En lo que se refiere a la industria pirotécnica, a nivel nacional la pirotecnia ha tenido un fuerte desarrollo durante los últimos años, dejando una derrama económica de 1,200 millones de pesos anuales (Ortega, 2018; Rincón, 2016; Ríos, 2018). De toda la producción nacional, se estima que el $70 \%$ proviene del Estado de México y el $50 \%$ proviene de Tultepec; lo cual deja unas ganancias de 400 a 600 millones de pesos para el municipio (Rincón, 2016; Ríos, 2018; Solís, 2018). Tultepec es uno de los 125 municipios del Estado de México localizado en la región XIV, en la porción nororiente de la entidad; y acuerdo con el Instituto Mexiquense de la Pirotecnia (IMEPI), comenzó con la producción de fuegos artificiales a principios del siglo XIX convirtiéndose en el pionero de la fabricación de cohetes en México y logrando el reconocimiento como "la capital de la pirotecnia". Por otra parte, el Departamento de Fomento Artesanal de Tultepec indica que la producción de fuegos artificiales es la principal actividad económica de la región, la cual llega a emplear hasta el $60 \%$ de la población en alguna de las actividades relacionadas con la comercialización de pirotecnia. En ese sentido, la producción de pirotecnia está altamente concentrada en Tultepec, por lo que su estudio puede resultar significativo para la comprensión de toda la industria. Asimismo, aunque no hay información precisa sobre el número total de empresas que participan en todo el sistema 
Villagómez Manrique, A. R. y Acosta Gonzaga, E. Uso de redes sociales digitales como

estrategia de mercadotecnia en pymes pirotécnicas de Tultepec en el estado de México

productivo de pirotecnia en Tultepec, en entrevistas a los medios de comunicación se ha revelado que existen en total 300 locales para la venta de juguetería pirotécnica ubicados en el mercado de San Pablito y 178 talleres autorizados para la fabricación de pirotecnia (Solís, 2018).

\subsection{Las redes sociales digitales y su relevancia para las pymes}

Las redes sociales digitales, también conocidas como social media o, simplemente, redes sociales, han generado una gran cantidad de investigación sobre las técnicas de implementación que utilizan las empresas y sobre los beneficios y usos que éstas obtienen al incluirlas como parte de sus estrategias. Sin embargo, el entendimiento intuitivo del término hizo que se prestara poca atención a la construcción de una definición uniforme y consistente. En ese contexto, Kaplan \& Haenlein (2010) propusieron una definición ampliamente aceptada dentro de la comunidad científica: "los medios sociales son un grupo de aplicaciones basadas en Internet construidas sobre los fundamentos ideológicos y tecnológicos de la Web 2.0 y que permiten la creación e intercambio de contenido generado por el usuario". Dentro de esta amplia definición podemos encontrar plataformas como Facebook, YouTube, Wikipedia y Pinterest, entre otras; cuyas características son diferentes y las hacen susceptibles de clasificación.

Una característica que tienen en común es que, recientemente, las organizaciones con y sin fines de lucro, de todos los sectores económicos y de las industrias primarias, secundarías y terciarias las han utilizado de muy diversas maneras como parte de su estrategia para incrementar su presencia en la sociedad (Chan \& Kumar, 2017; Crammond, Omeihe, Murray, \& Ledger, 2018; Waters, Burnett, Lamm, \& Lucas, 2009). Sin embargo, a menudo las organizaciones consideran las redes sociales como elementos individuales que operan independientemente (Hanna, Rohm, \& Crittenden, 2011); mientras que en la literatura científica se propone que las redes sociales deben ser consideradas como parte de un ecosistema de elementos interrelacionados, que involucran tanto a medios tradicionales como medios digitales (Hanna et al., 2011), para aprovechar al máximo el potencial que tienen para apoyar los objetivos estratégicos de la empresa.

Otro aspecto que refuerza la necesidad del estudio de las redes sociales digitales es el desarrollo de la Web 2.0, que permitió que los consumidores adoptaran un rol más activo en los procesos de intercambio de mercadotecnia; pasando ser meros receptores de información hasta convertirse en una extensión de la organización capaz de crear contenido, diseñar nuevo productos o servicios o producir mensajes promocionales (Hanna et al., 2011). Esto es, el consumidor moderno busca conectar con las empresas a las que les compra y con otros consumidores que puedan ofrecer información relevante (Garretson, 2008). En este nuevo ecosistema, la mercadotecnia ya no puede enfocarse solamente en captar la atención de la sociedad, sino que también debe contar con estrategias que permitan retener su atención y conectar con ella. Por lo cual, el uso de redes sociales digitales para fortalecer los vínculos entre la organización y la sociedad se ha convertido en una práctica esencial. 
Villagómez Manrique, A. R. y Acosta Gonzaga, E. Uso de redes sociales digitales como

estrategia de mercadotecnia en pymes pirotécnicas de Tultepec en el estado de México

\subsection{Tendencias en las investigaciones sobre redes sociales digitales en pymes}

Existe una amplia literatura sobre los usos que dan las pymes de diversos sectores a las redes sociales, entre los cuales se encuentran: alcanzar sus objetivos de mercadotecnia, atraer nuevos clientes, cultivar las relaciones con los clientes actuales, posicionar sus productos mediante la publicidad boca a boca, alcanzar amplias audiencias (Ainin, Parveen, Moghavvemi, Jaafar, \& Shuib, 2015), estimar la percepción de los clientes sobre nuevos productos y promociones, recibir retroalimentación de los clientes (Wamba \& Carter, 2014) y para buscar innovaciones en sus productos o servicios (Scuotto, Del Giudice, Peruta, \& Tarba, 2017).

Adicionalmente, las investigaciones realizadas detectan diversos beneficios a partir del uso de redes sociales digitales como: mejora de la administración del conocimiento en tiempo real entre agentes tanto internos como externos a la organización (Crammond et al., 2018), impulso del crecimiento del capital social, el cual conduce a un conocimiento organizacional de mayor calidad; reducción de la incertidumbre en las inversiones relacionadas con la innovación de productos o servicios (Scuotto et al., 2017) y mejora en el proceso de internacionalización (Zhou, Wu, \& Luo, 2007), por mencionar algunos.

Por otro lado, los académicos han concentrado su atención en determinar los elementos que deben considerarse para una adecuada adopción de redes sociales (Kaplan \& Haenlein, 2010; Lashgari, Sutton-Brady, Solberg Søilen, \& Ulfvengren, 2018) y los factores que influyen en la adopción de redes sociales (Ainin et al., 2015; BraojosGomez, Benitez-Amado, \& Javier Llorens-Montes, 2015; Wamba \& Carter, 2014).

A pesar de esta basta cantidad de información, hay muy pocos estudios sobre el uso de tecnología digital en pymes para alcanzar objetivos relacionados con la mercadotecnia (e-marketing o marketing digital) y aún menos, sobre el uso y adopción de redes sociales en pymes mexicanas (Cota \& Antonio, 2016; GutierrezLeefmans, Nava-Rogel, \& Trujillo-Leon, 2016). Así, la exposición anterior hace evidente la necesidad de realizar más estudios empíricos sobre el uso de RSD en pymes $y$, principalmente, en las pymes de México, las cuales tienen tanta preponderancia en la economía nacional. Por lo tanto, el presente trabajo pretende estudiar el uso y adopción de RSD en las pymes de la industria de la pirotecnia en México, cuyos resultados servirán: a las pymes para conocer los beneficios que se obtienen de su implementación y su uso por parte de la competencia; y al gobierno local, para la creación de campañas de difusión y capacitación en redes sociales digitales con propósitos empresariales.

\section{OBJETIVOS}

A pesar de la relevancia de las pymes para México, no hay suficiente literatura científica sobre el nivel de uso de las redes sociales digitales en las pymes mexicanas. Por ello, el objetivo de esta investigación es determinar el grado de 
Villagómez Manrique, A. R. y Acosta Gonzaga, E. Uso de redes sociales digitales como

estrategia de mercadotecnia en pymes pirotécnicas de Tultepec en el estado de México

adopción de RSD y su empleo como estrategia de mercadotecnia en las pymes de la industria pirotécnica de Tultepec, Estado de México.

\section{METODOLOGÍA}

Para cumplir con el objetivo de esta investigación, se seleccionaron aquellos agentes cuya principal actividad económica, al momento de realizar las encuestas, estuviera directamente relacionada con la pirotecnia (fabricación, comercialización, o alguna otra actividad relacionada o auxiliar) y con ubicación dentro del municipio de Tultepec. Se aplicaron un total de 20 cuestionarios a los dueños de las empresas pirotécnicas de Tultepec; tales cuestionarios se aplicaron en los meses de octubre 2018 a enero 2019 en tres ubicaciones principales dentro del municipio: el mercado de cohetes de "San Pablito", la zona de talleres de "La Saucera" y en las tiendas de materias.

El instrumento de recolección de datos se basa en el cuestionario utilizado en (Cota \& Antonio, 2016), el cual se usó para determinar el porcentaje de empresas en Guadalajara que han usado redes sociales digitales, así como los beneficios que esta estrategia les ha traído. El cuestionario consta de tres partes: 1 ) datos generales, 2) adopción y usos y 3) beneficios (ver anexo). La sección 2 (adopción y usos) fue adaptada para las pymes de la industria pirotécnica. Adicionalmente, se creó una tabla de usos de RSD, a partir de la clasificación de los principales recursos digitales que dan soporte al marketing mix de 7p's propuesto por (Gutierrez-Leefmans et al., 2016). El marketing mix es el conjunto de elementos fundamentales que dirigen las estrategias de mercadotecnia (Gutierrez-Leefmans et al., 2016). Inicialmente, el marketing mix se componía de cuatro elementos: price (precio), product (producto), place (plaza) y promotion (promoción) (Pereault \& McCarthy, 2002), posteriormente, la teoría evolucionó hasta llegar a las 7 p's: price, product, place, promotion, people (personas), process (proceso) y physical evidence (evidencia física) (Booms \& Bitner, 1981). Con base en este conjunto de variables, Chaffey (2015) añadió los recursos digitales que corresponden a cada variable de las 7 p's, como se muestra en la siguiente tabla.

Tabla 1. Principales recursos digitales que dan soporte al marketing mix. Modificado de Gutiérrez-Leefmans et al. (2016).

\begin{tabular}{|c|c|}
$\begin{array}{c}\text { Elemento del } \\
\text { marketing mix }\end{array}$ & \multicolumn{1}{c}{ Principales recursos digitales que dan soporte a cada elemento } \\
\hline Producto & $\begin{array}{l}\text { Producto principal y producto extendido (avales, premios, testimonios, listas de } \\
\text { clientes, comentarios, garantías, ofertas de devolución de dinero, servicio al } \\
\text { cliente y recursos para ayudar a los clientes a usar el producto). }\end{array}$ \\
\hline Precio & $\begin{array}{l}\text { Políticas de precios en línea que pueden incluir: precios básicos, descuentos, } \\
\text { garantías, políticas de reembolso, términos de cancelación, complementos y } \\
\text { productos o servicios adicionales. }\end{array}$ \\
\hline Plaza & $\begin{array}{l}\text { Los canales de Internet pueden ser solo un canal de comunicación, un canal de } \\
\text { distribución a intermediarios o un canal de venta directa a los consumidores. }\end{array}$ \\
\hline
\end{tabular}


Villagómez Manrique, A. R. y Acosta Gonzaga, E. Uso de redes sociales digitales como

estrategia de mercadotecnia en pymes pirotécnicas de Tultepec en el estado de México

\begin{tabular}{|c|c|}
\hline Promoción & $\begin{array}{l}\text { Publicidad (anuncios gráficos interactivos, motores de búsqueda); ventas (chat, } \\
\text { agentes virtuales); promoción de ventas (cupones, descuentos, programas de } \\
\text { fidelización); relaciones públicas (blogs, boletines, redes sociales); patrocinios en } \\
\text { línea; correo directo (boletines y correos electrónicos personalizados); } \\
\text { exposiciones virtuales; comercialización (recomendaciones personalizadas); } \\
\text { embalaje (embalaje que se muestra en línea); boca a boca (marketing viral). }\end{array}$ \\
\hline Personas & $\begin{array}{l}\text { Acciones que pueden automatizarse: respuestas automáticas, notificaciones por } \\
\text { correo electrónico, servicio de devolución de llamada, preguntas frecuentes } \\
\text { (FAQ, por sus siglas en inglés), asistentes virtuales. }\end{array}$ \\
\hline Procesos & $\begin{array}{l}\text { Métodos y procesos en la oficina de apoyo (back-office) para lograr las funciones } \\
\text { de marketing. }\end{array}$ \\
\hline Evidencia física & Sitio centrado en el usuario, empaque, política de privacidad. \\
\hline
\end{tabular}

A partir de la tabla anterior, se construyó una tabla de usos (tabla 2) para determinar el uso que las pymes pirotécnicas hacen de las redes sociales digitales y clasificar tales usos de acuerdo con el soporte que brindan al marketing mix de 7 p's, mediante una escala de frecuencia de uso.

Para el análisis de los datos se utilizó la estadística descriptiva y como método de validación de los datos resultados obtenidos se utilizó la saturación teórica (Denzin \& Lincoln, 2005) para determinar el momento de concluir la recolección de datos. Además, del riesgo de una posible explosión, acción propia de la actividad económica pirotécnica, fue otro de los factores considerados para concluir el proceso de recolección.

\section{RESULTADOS Y DISCUSIÓN}

Los resultados de la aplicación de 20 cuestionarios a los diferentes agentes que intervienen en el encadenamiento productivo de pirotecnia se muestran a continuación: Los encuestados se clasifican como proveedores de materias primas $(10 \%)$, fabricantes $(55 \%)$, mayoristas $(20 \%)$ y minoristas (15\%). Respecto al tamaño de las empresas, el número de empleados que trabajan de estas empresas corresponde a la categoría de micro $(75 \%)$ y pequeñas empresas $(25 \%)$.

Respecto a las redes sociales digitales, el $85 \%$ de los encuestados respondieron que no se encuentran inscritos en ninguna red social digital y sólo $15 \%$ afirmaron usar alguna RSD (ver gráfica 1). Esto ejemplifica el estado incipiente del nivel de uso de las redes sociales como una estrategia de marketing en dicha industria mexicana. 


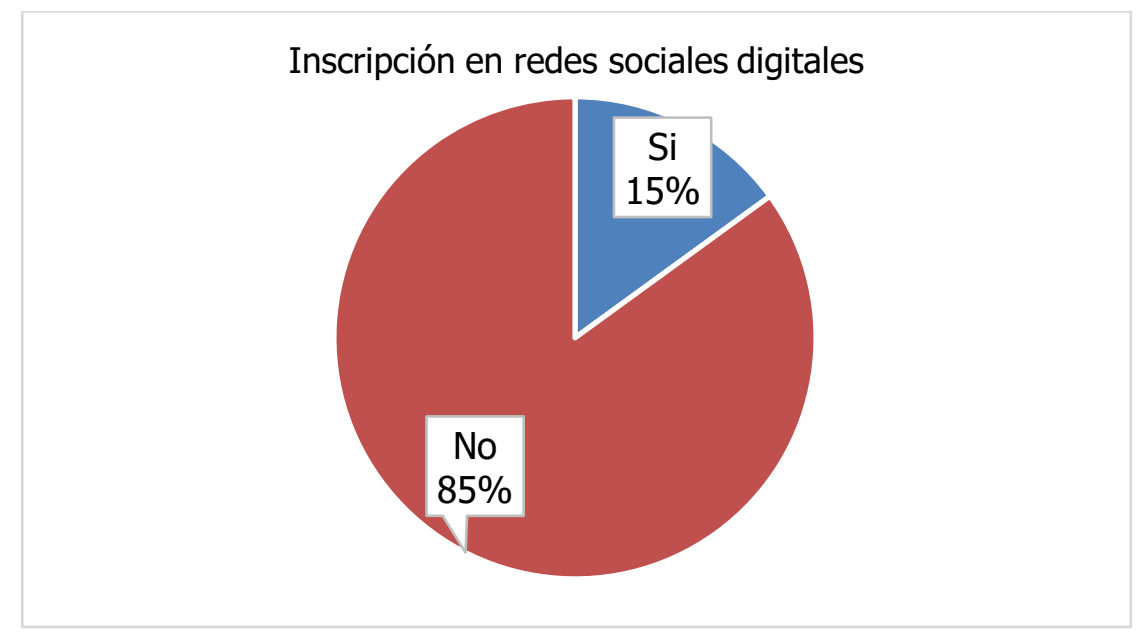

Gráfica 1: inscripción en redes sociales de las empresas pirotécnicas en Tultepec. Fuente: elaboración propia.

Para las empresas que afirmaron el uso de alguna RSD, la red social más utilizada es Facebook (33\%) seguida por YouTube (22\%), aunque también usan otro tipo de redes como Twitter e Instagram (ver gráfica 2). Además, se encontró que el $22 \%$ de las empresas usan, portales o plataformas Web. Los encuestados no mencionaron alguna otra red, lo que significa que se mantienen en las redes sociales más populares.

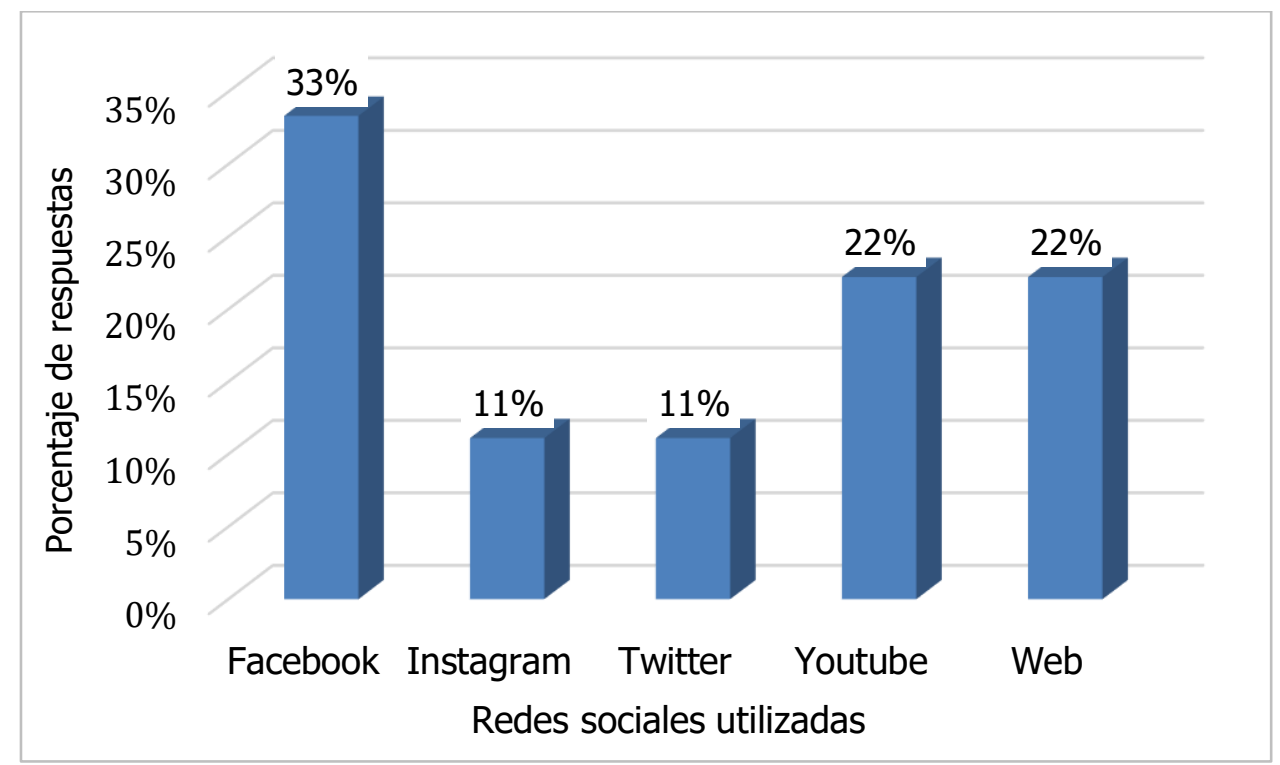

Gráfica 2: principales redes sociales utilizadas por las empresas de pirotecnia en Tultepec.

Fuente: elaboración propia.

Respecto a la cantidad de tiempo en el uso de los medios sociales, los resultados (ver gráfica 3) muestran que, en algunos casos se han utilizado desde hace más de 10 años, mientras que, en otros, las usan recientemente. Además, respecto a la frecuencia con que las utilizan (ver gráfica 4): $67 \%$ de las empresas reconocen 
usarlas diariamente, mientras que el 33\% afirma utilizarlas sólo en la época de alta demanda (septiembre-diciembre). Tal hallazgo sugiere que los empresarios reconocen la importancia de dar seguimiento a las actividades en redes sociales.

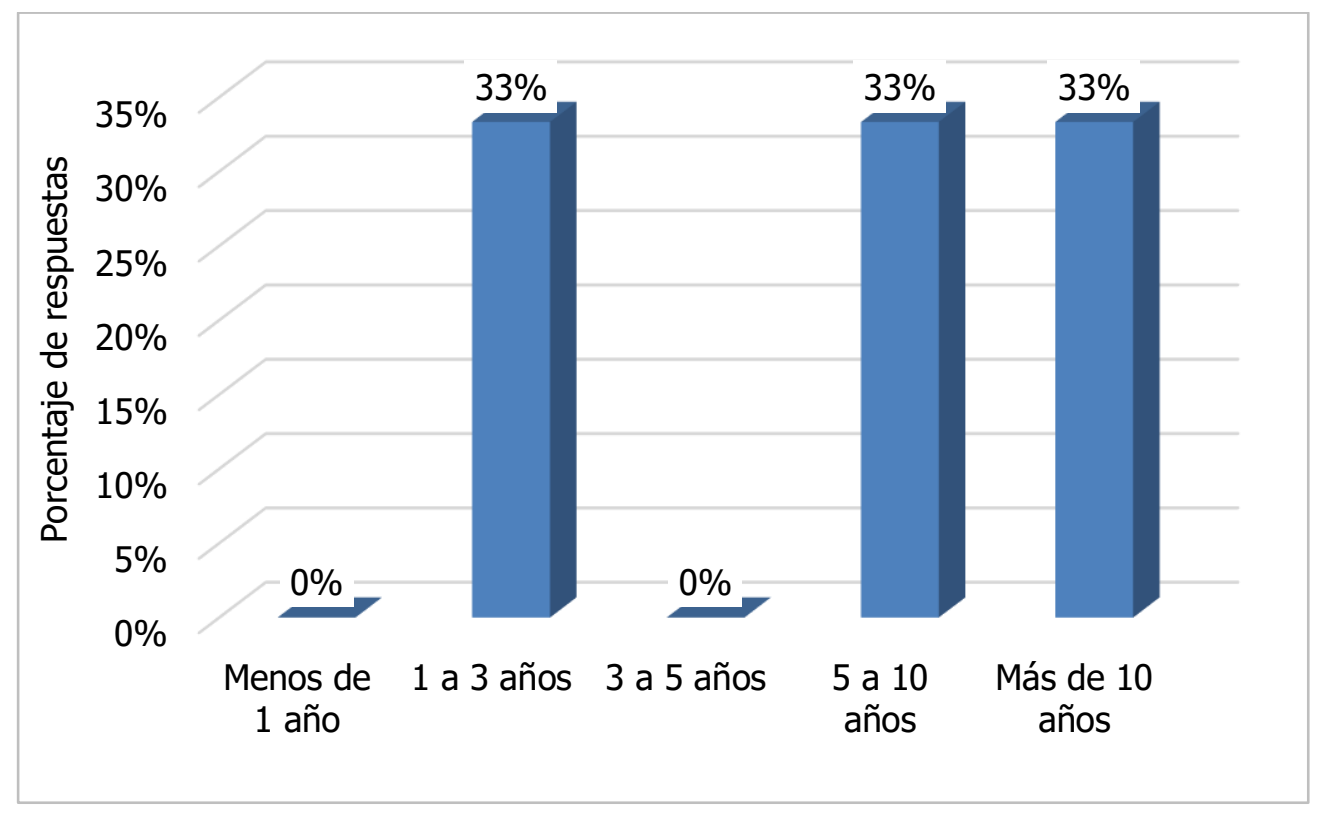

Gráfica 3: número de años usando redes sociales digitales.

Fuente: elaboración propia.

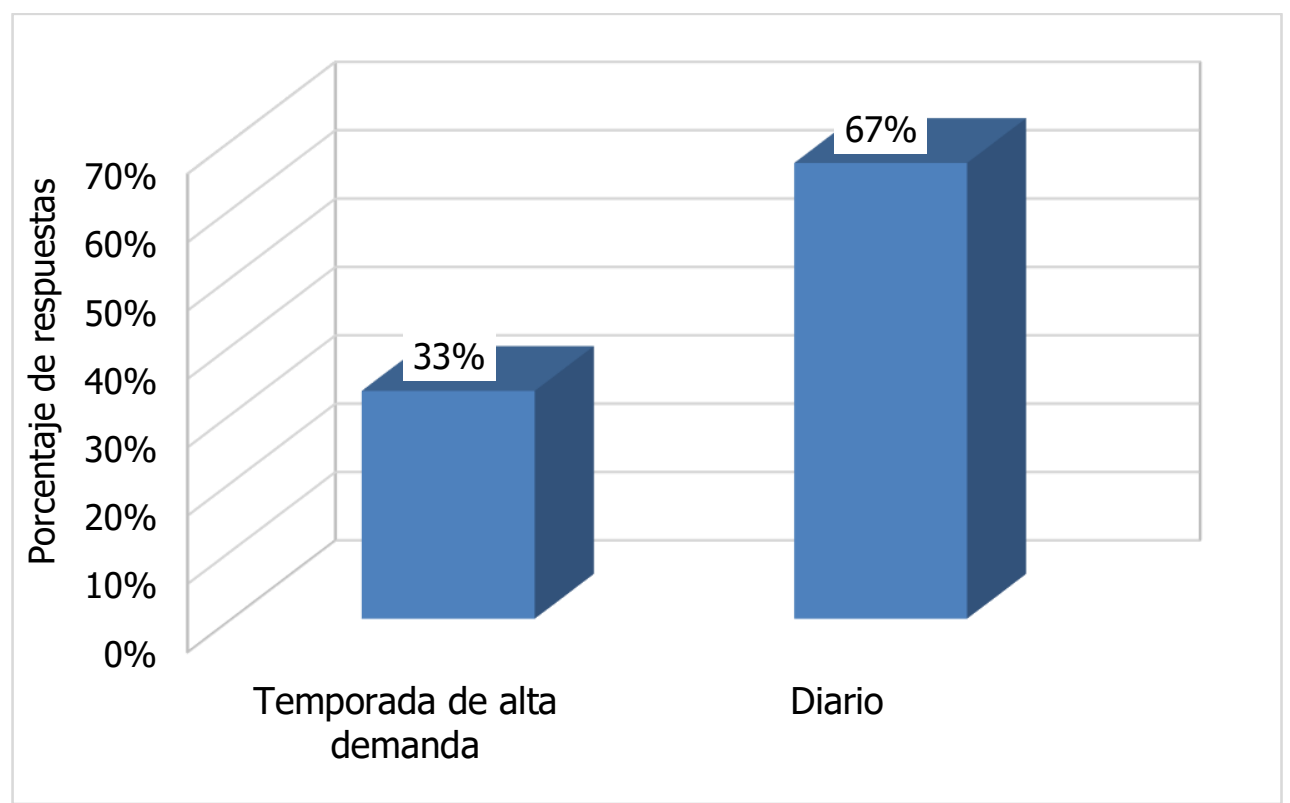

Gráfica 4: frecuencia de uso de redes sociales digitales.

Fuente: elaboración propia.

Si bien algunas pymes utilizan las RSD como estrategia de marketing, los empresarios coinciden en que las redes sociales no se administran adecuadamente por una persona con formación especializada (community manager), sino que en la 
mayoría de los casos es administrada por un familiar (gráficas 5 y 6 ). Lo cual implica que no hay (o cuando menos es poco probable) que exista una planeación de la estrategia de marketing a implementar, que contemple métricas específicas que ayuden a determinar de manera confiable el impacto del uso de las redes sociales. Esto, refuerza lo planteado por (Becker \& Lee, 2019) quienes argumentan que las empresas más grandes hacen un uso más efectivo de sus redes sociales.

\section{CONTRATACIÓN DE PERSONAL ESPECIALIZADO}

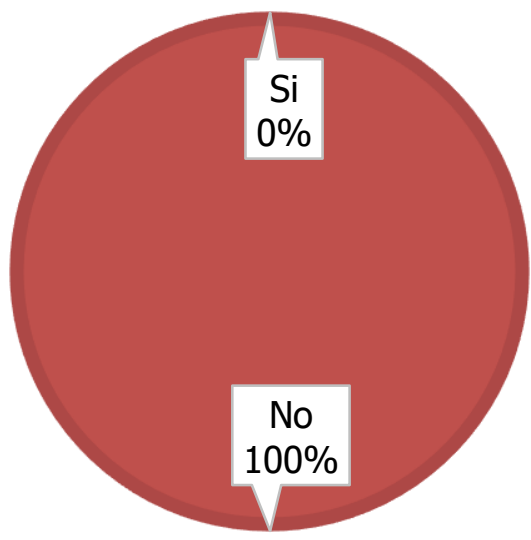

Gráfica 5: formación del personal encargado de las redes sociales digitales.

Fuente: elaboración propia.

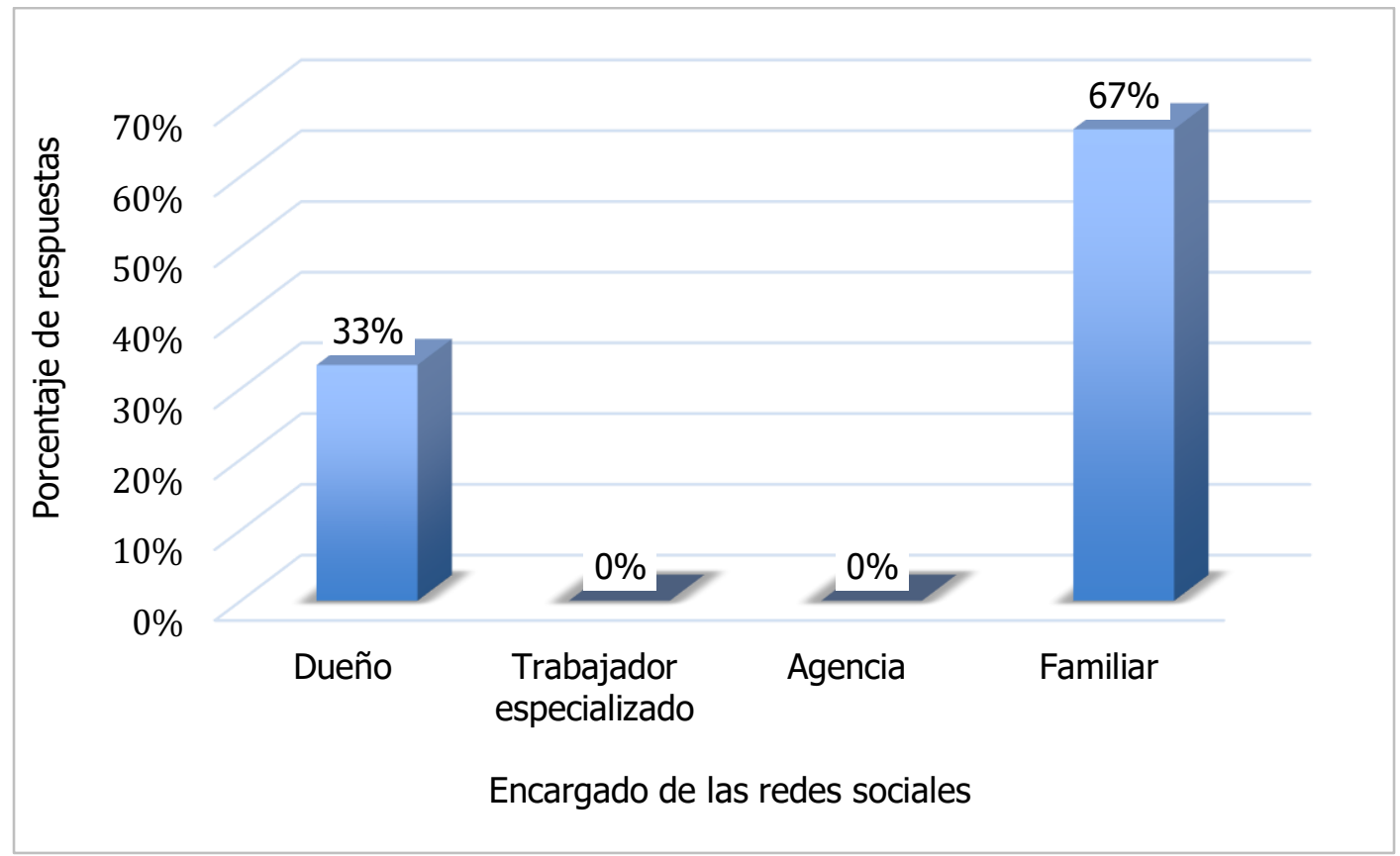

Gráfica 6: personal encargado de las redes sociales digitales.

Fuente: elaboración propia. 
Villagómez Manrique, A. R. y Acosta Gonzaga, E. Uso de redes sociales digitales como

estrategia de mercadotecnia en pymes pirotécnicas de Tultepec en el estado de México

Por otro lado, los usos que las empresas pirotécnicas han dado a RSD se resumen en la siguiente tabla:

Tabla 2. Usos de las redes sociales digitales por parte de las empresas pirotécnicas.

\begin{tabular}{|l|c|c|c|}
\hline \multicolumn{1}{|c|}{ Uso } & $\begin{array}{c}\text { Nunca/poco } \\
\text { frecuente }\end{array}$ & Frecuente & $\begin{array}{c}\text { Siempre/muy } \\
\text { frecuente }\end{array}$ \\
\hline $\begin{array}{l}\text { Proporcionar información sobre el uso de los } \\
\text { productos }\end{array}$ & $67 \%$ & $33 \%$ & $0 \%$ \\
\hline $\begin{array}{l}\text { Proporcionar información de las características de los } \\
\text { productos }\end{array}$ & $33 \%$ & $33 \%$ & $33 \%$ \\
\hline Dar descuentos en los productos & $67 \%$ & $33 \%$ & $0 \%$ \\
\hline $\begin{array}{l}\text { Proporcionar información de los precios de los } \\
\text { productos }\end{array}$ & $0 \%$ & $33 \%$ & $67 \%$ \\
\hline Vender a intermediarios & $33 \%$ & $67 \%$ & $0 \%$ \\
\hline Vender a clientes finales/consumidores & $0 \%$ & $67 \%$ & $33 \%$ \\
\hline Dar demostraciones virtuales de los productos & $67 \%$ & $0 \%$ & $33 \%$ \\
\hline Mejorar sus relaciones con sus clientes & $33 \%$ & $33 \%$ & $33 \%$ \\
\hline Anunciar eventos & $33 \%$ & $67 \%$ & $0 \%$ \\
\hline Dar asistencia en línea & $100 \%$ & $0 \%$ & $0 \%$ \\
\hline Recibir opiniones sobre los productos/servicios & $0 \%$ & $67 \%$ & $33 \%$ \\
\hline
\end{tabular}

Fuente: elaboración propia a partir de la clasificación de 7p's de los recursos digitales.

La tabla 2 resume los usos que las empresas pueden dar a las redes sociales para conseguir sus objetivos de marketing, tales usos se determinaron a partir de la clasificación de 7p's de los recursos digitales (Gutierrez-Leefmans et al., 2016). Los colores ayudan a identificar visualmente el grado de consenso de las respuestas de acuerdo con lo siguiente: los recuadros marcados con color amarillo indican que hay un acuerdo parcial en las respuestas obtenidas, es decir, indican que una fracción de los encuestados proporcionaron la misma respuesta. Por otro lado, los recuadros marcados con color verde indican un acuerdo total, esto es, que todos los encuestados respondieron de la misma forma al reactivo.

De acuerdo con la tabla 2, el $67 \%$ de las empresas pirotécnicas que utilizan redes sociales como estrategia digital no usan las redes sociales para proporcionar información sobre el uso de sus productos, ni para ofrecer descuentos, ni para dar demostraciones virtuales de sus productos. Además, se encontró que $100 \%$ de las pymes que usan RSD coinciden en que no las utilizan para proporcionar asistencia en línea sobre sus productos.

Asimismo, el $67 \%$ de los encuestados que utilizan RSD afirmaron que frecuentemente las utilizan para: vender a intermediarios, vender a clientes finales 0 consumidores, anunciar eventos o recibir opiniones sobre los productos/servicios (McCann \& Barlow, 2015; Odoom, Anning-dorson, \& Acheampong, 2017; Yao, Boyer, \& Baker, 2018). Finalmente, la tabla 2 muestra que el $67 \%$ de las pymes utilizan principalmente sus redes sociales para dar información sobre los precios de los productos o servicios. 
Se encontró que los usos principales que dan los empresarios pirotécnicos a las redes sociales son: para posicionar el producto y alcanzar mayores audiencias (Ainin et al., 2015; Odoom et al., 2017; Yao et al., 2018) y para recibir retroalimentación de los clientes (Wamba \& Carter, 2014).

Además, los encuestados reconocen que el uso de redes sociales ha traído beneficios. La gráfica 7 muestra que los beneficios obtenidos son: una popularidad mayor para la marca $(43 \%)$, incremento en las ventas (29\%), aumento en la cantidad de trabajo (14\%) y mejor relación con los clientes (14\%). Estos resultados indican que los beneficios obtenidos están alineados con los usos que los empresarios dan a las redes sociales digitales (mostrados en la tabla 2): posicionar el producto y recibir retroalimentación.

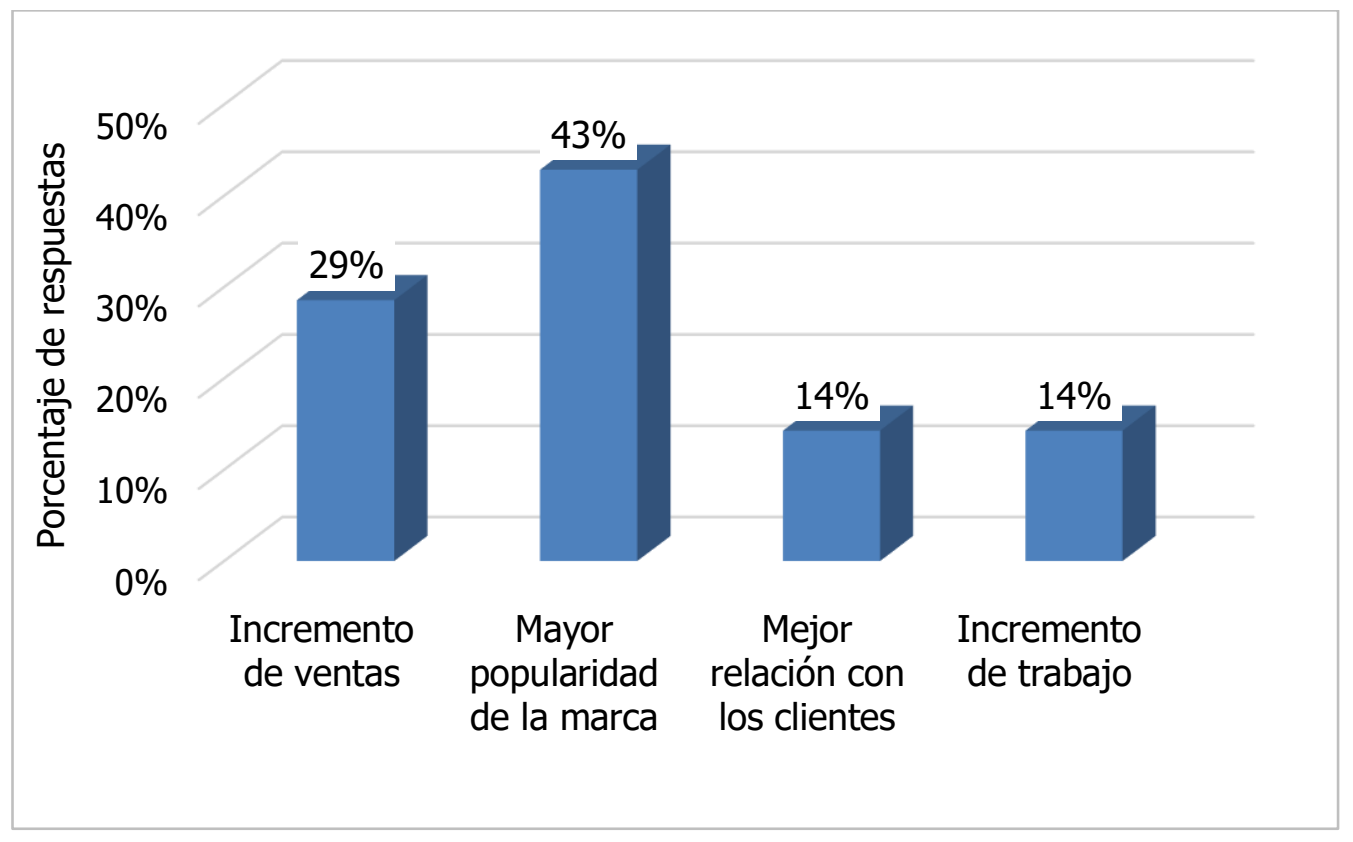

Gráfica 7: beneficios del uso de redes sociales.

Fuente: elaboración propia.

Respecto a la obtención de clientes nuevos, la gráfica 8 muestra que el $67 \%$ de los informantes afirma haber conseguido clientes nuevos mediante el uso de redes sociales digitales. Con lo anterior, se refuerzan los resultados de investigaciones previas que afirman que el uso de RSD como estrategia de marketing puede resultar en un incremento de las ventas y de las ganancias para las pymes (Yao et al., 2018). Como se puede observar en la gráfica 9 , los clientes nuevos son de otros municipios del estado (50\%) y de otros estados de la república (50\%). Esto se explica con el hecho de que, el sistema productivo de pirotecnia de Tultepec está enfocado en los mercados localizados fuera del municipio y, por lo tanto, el propio municipio no es un consumidor de sus productos (Villagómez-Manrique, 2019). Por otro lado, la exportación de pirotécnica está fuertemente regulada por una gran cantidad de países, de modo que resulta difícil llegar a los mercados internacionales y por ello, es difícil obtener clientes extranjeros. 
Villagómez Manrique, A. R. y Acosta Gonzaga, E. Uso de redes sociales digitales como estrategia de mercadotecnia en pymes pirotécnicas de Tultepec en el estado de México

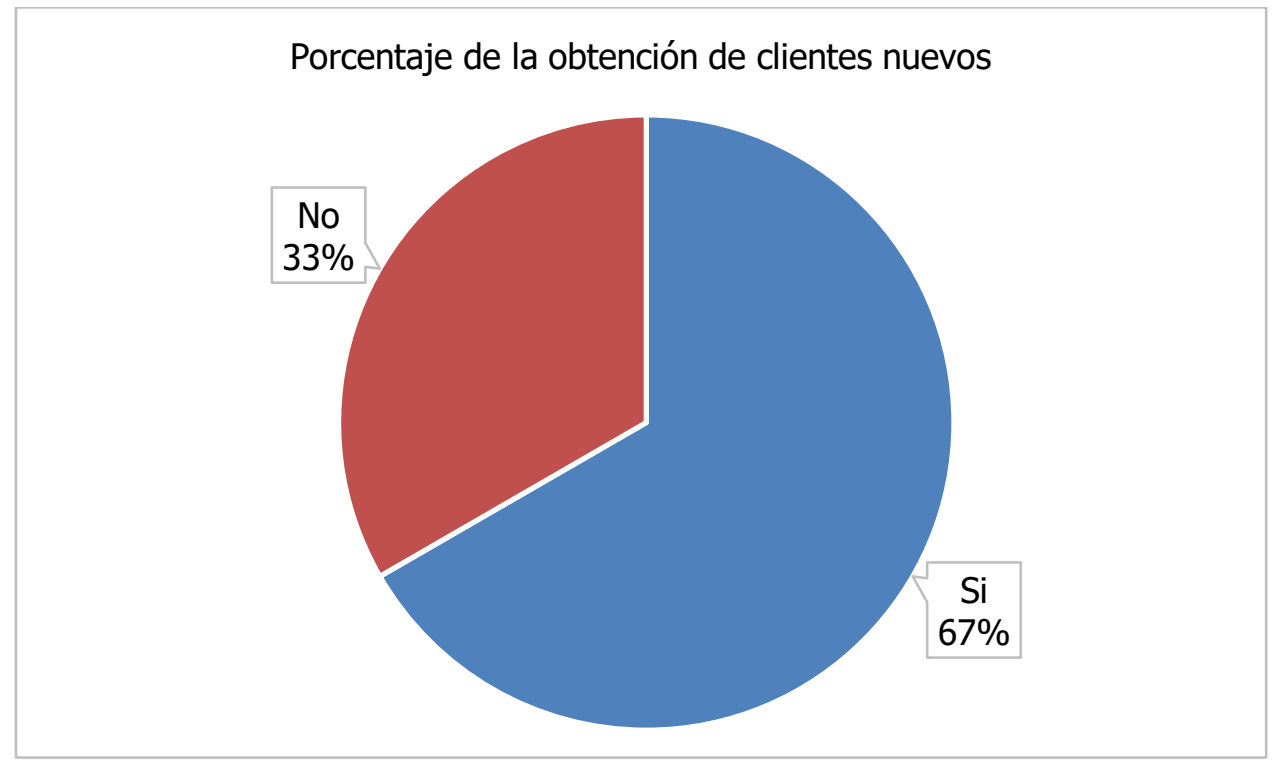

Gráfica 8: obtención de clientes nuevos mediante el uso de redes sociales digitales. Fuente: elaboración propia.

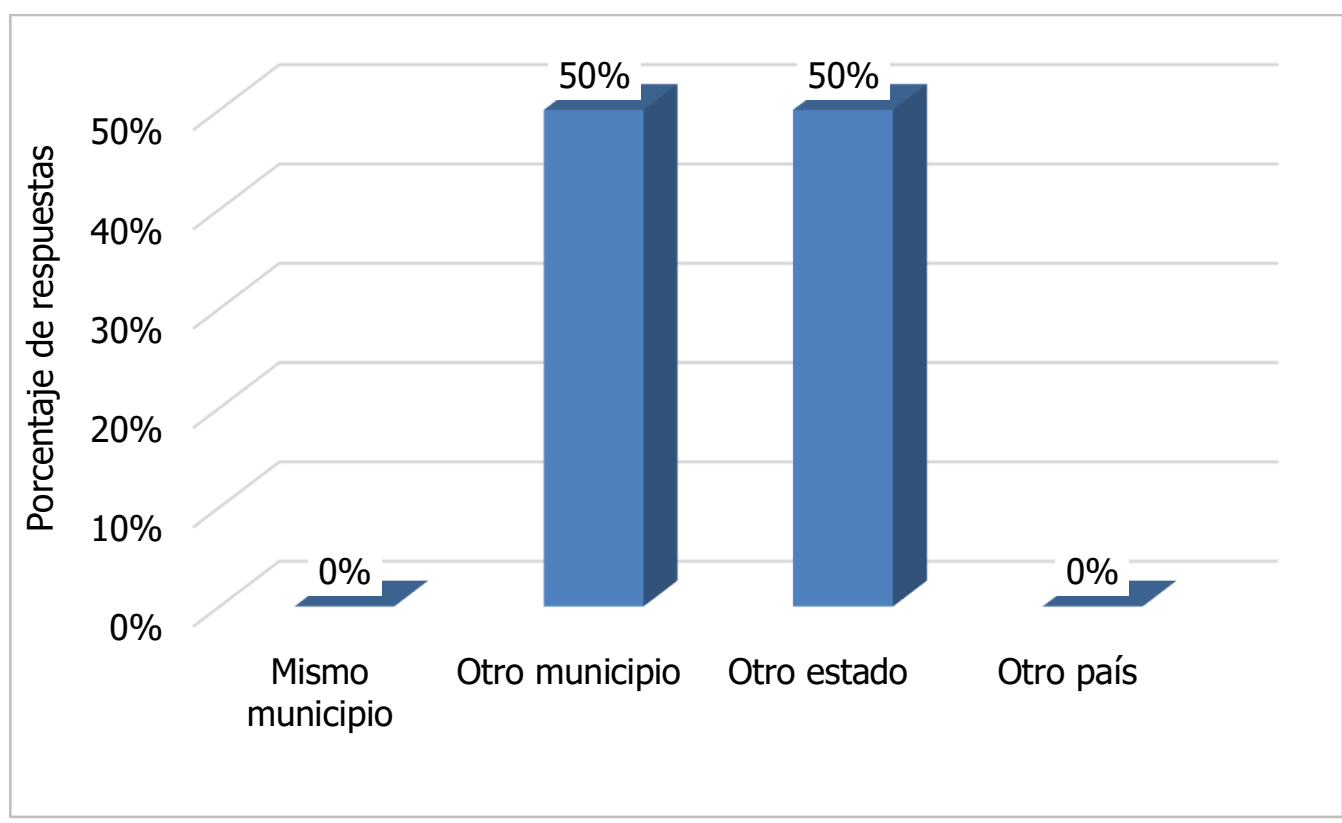

Gráfica 9: procedencia de los clientes nuevos adquiridos a través de RSD. Fuente: elaboración propia.

En lo que refiere al uso general de redes sociales, el $100 \%$ de las pymes que las utilizan también las recomiendan y las razones principales que mencionan para recomendarlas son: "es más fácil que aparezcan anuncios a las personas", "porque todo el mundo está conectado" y porque "sí se consiguen más clientes". Por otro lado, el $85 \%$ de los encuestados mencionaron las razones para no utilizar las redes sociales digitales, las cuales se pueden resumir en tres categorías (ver gráfica 10): 1) consideran que están bien posicionados y por lo tanto, no necesitan usar las RSD, 2) no saben usar las RSD y, 3) han tenido problemas relacionados con la delincuencia y 
la inseguridad. Esto se puede observar por los comentarios que algunos empresarios mencionaron: "muchas veces, nada más te hablan por molestar o son extorsiones", "tuve problemas con la delincuencia recientemente" y "es muy peligroso"; los encuestados mencionaron las expresiones anteriores, como resultado de su experiencia en el uso de redes sociales digitales como estrategia de mercadotecnia. Dichas experiencias pueden incluir asalto, extorsión y en algunos casos hasta secuestro.

Con relación a la inseguridad en RSD, se ha encontrado que uso de redes sociales puede implicar diversos riesgos, ya que los criminales pueden utilizarlas para: cometer fraude, robar información sensible, el robo de identidad, realizar ciber espionaje y cometer actos de venganza, entre otros (He, 2012; Rose, 2011; Tayouri, 2015; Zhang \& Gupta, 2018). Si bien Facebook es la red social más popular, también es la RSD que reporta mayor número de riesgos de seguridad, adjudicándose el $61 \%$ de los casos (Zhang \& Gupta, 2018). Así, compartir demasiada información en las redes sociales digitales, las cuales a su vez comparten dicha información con terceros, hace posible un mal uso de esa información y puede crear riesgos de seguridad (Rose, 2011).

Por consiguiente, es importante realizar una adecuada difusión de los beneficios que se pueden obtener del uso de redes sociales digitales como parte de una estrategia de marketing. Adicionalmente, es esencial capacitar a la población para hacer un uso responsable de dichas RSD y así evitar compartir información innecesaria que pueda ser utilizada con la intención de causar daño. Sin embargo, además de la capacitación se recomienda utilizar herramientas tecnológicas que ayuden a administrar el flujo de información en RSD y que puedan detectar y reparar vulnerabilidades de seguridad (Tayouri, 2015).

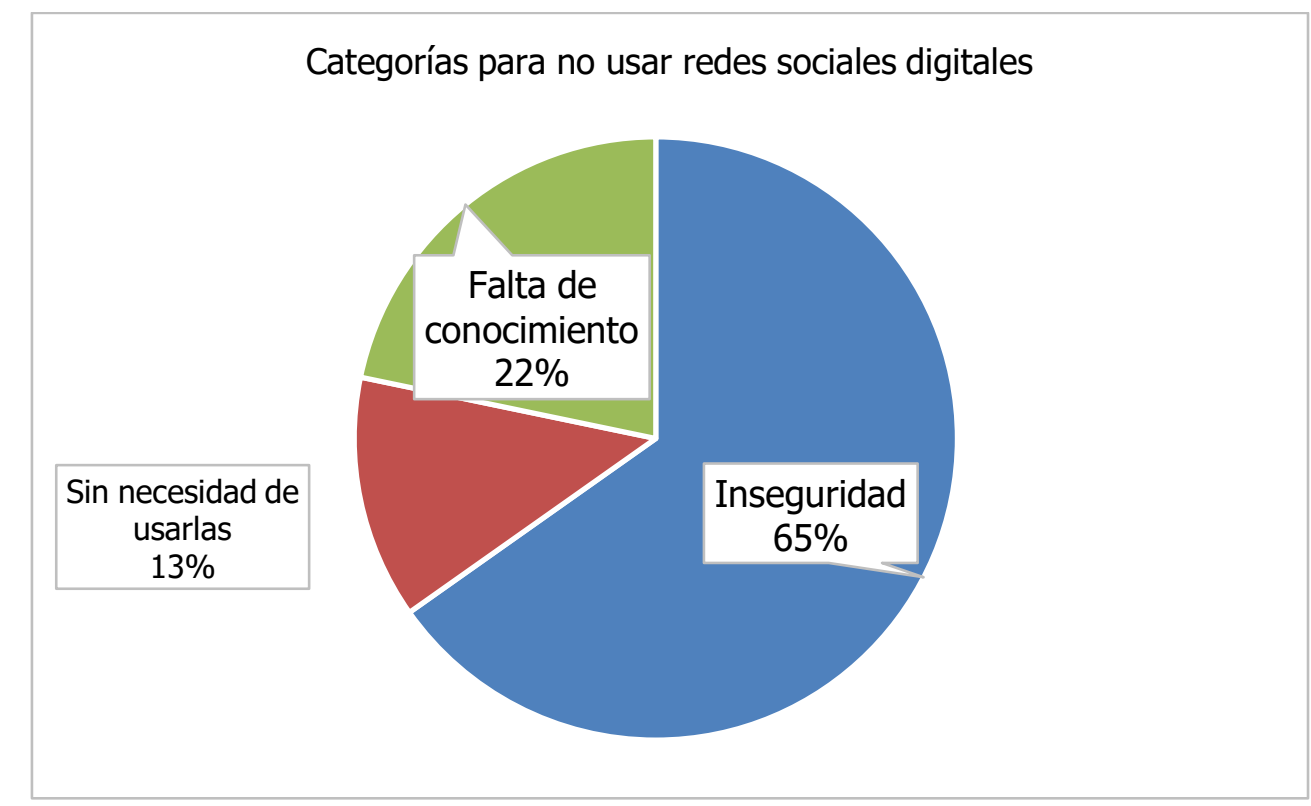

Gráfica 10: principales razones para no utilizar redes sociales digitales.

Fuente: elaboración propia. 
Villagómez Manrique, A. R. y Acosta Gonzaga, E. Uso de redes sociales digitales como

estrategia de mercadotecnia en pymes pirotécnicas de Tultepec en el estado de México

\section{CONCLUSIONES}

Como resultado de la investigación, se puede concluir que la tabla de usos de redes sociales digitales elaborada con base en la clasificación de 7 p's (GutierrezLeefmans et al., 2016) resultó un buen instrumento para la clasificación de los usos de las redes sociales digitales y el desarrollo de este instrumento en futuras investigaciones podría conducir a una herramienta estandarizada para que sirva para una clasificación más exhaustiva y actualizada.

Por otro lado, el nivel de uso de redes sociales en el sistema productivo de pirotecnia en Tultepec es, en general, limitado. Sólo el $15 \%$ de los encuestados las emplean como parte de su estrategia de marketing y se limitan a utilizar las redes sociales más conocidas: Facebook, YouTube y sitios web. No contratan personal especializado para manejar sus redes, si no que las maneja algún familiar (principalmente los hijos).

Los usos que dan los empresarios pirotécnicos a las redes sociales digitales son aquellos orientados al posicionamiento del producto, a alcanzar mayores audiencias y a recibir retroalimentación de los clientes. En consecuencia, los principales beneficios recibidos son aumento en las ventas y mejora en la relación con los clientes. Por ello, todos recomiendan su uso como parte de la estrategia de negocio.

En contraste, el $85 \%$ de los encuestados no utiliza redes sociales digitales. Las razones que mencionaron son: inseguridad principalmente, desconocimiento de su uso y porque consideran estar bien posicionados y no necesitarlas. Lo anterior refleja, en primer lugar, una necesidad de difundir los beneficios que las redes sociales digitales pueden traer a las pymes mexicanas y la forma más efectiva de usarlas para alcanzar tales beneficios; $y$, además, que existen grupos propensos a ser objetivos del crimen a causa de las vulnerabilidades en las redes sociales, que necesitan capacitación en el uso adecuado de herramientas digitales. El uso inadecuado de RSD puede ser aprovechado para cometer diferentes clases de crímenes, de modo que los empresarios se rehúsan a utilizarlas, desechando los posibles beneficios de implementarlas como parte de su estrategia de marketing.

Se espera que el presente trabajo motive a realizar investigaciones futuras que amplíen el conocimiento del uso de redes sociales digitales como estrategia de mercadotecnia en las pymes. Particularmente, resulta necesaria la investigación sobre las métricas que utilizan las pymes mexicanas para medir el impacto del uso de redes sociales y los factores que motivan su implementación como parte de la estrategia empresarial.

\section{Agradecimientos}

Esta investigación fue realizada con el apoyo del Consejo Nacional de Ciencia y Tecnología, a través de la beca otorgada a los estudiantes del programa de la Maestría en Ciencias en Estudios Interdisciplinarios en pequeñas y medianas empresas. También, agradecemos al Instituto Politécnico Nacional por proveer los recursos necesarios para esta investigación. 
Villagómez Manrique, A. R. y Acosta Gonzaga, E. Uso de redes sociales digitales como

estrategia de mercadotecnia en pymes pirotécnicas de Tultepec en el estado de México

\section{REFERENCIAS}

Ainin, S., Parveen, F., Moghavvemi, S., Jaafar, N. I., \& Shuib, N. L. M. (2015). Factors influencing the use of social media by SMEs and its performance outcomes. Industrial Management and Data Systems, 115(3), 570-588. doi: https://doi.org/10.1108/IMDS-07-2014-0205

Becker, K., \& Lee, J. W. (2019). Organizational Usage of Social Media for Corporate Reputation Management. The Journal of Asian Finance, Economics and Business, 6(1), 231-240.

doi: https://doi.org/10.13106/jafeb.2019.vol6.no1.231

Booms, B. H., \& Bitner, M. J. (1981). Marketing strategies and organization structures for service firms. Marketing of Services, 25(3), 47-52.

Braojos-Gomez, J., Benitez-Amado, J., \& Javier Llorens-Montes, F. (2015). How do small firms learn to develop a social media competence? International Journal of Information Management, 35(4), 443-458.

doi: https://doi.org/10.1016/j.ijinfomgt.2015.04.003

Chaffey, D. (2015). Digital Business \& E-Commerce Management (6th ed.). Pearson.

Chan, D., \& Kumar, S. (2017). Social Media Strategies for Small and Medium Scale Enterprise in the Klang Valley Region of Malaysia. In Handbook of Research on Small and Medium Enterprises in Developing Countries (pp. 377-400). doi: https://doi.org/10.4018/978-1-5225-2165-5.ch018

Cota, R., \& Antonio, J. (2016). Adopción de redes sociales digitales en las pymes de la industria del calzado de la zona metropolitana de Guadalajara Jalisco, México. Revista de Comunicación de La SEECI, (40), 48-68. doi: https://doi.org/10.15198/seeci.2016.40.48-68

Crammond, R., Omeihe, K. O., Murray, A., \& Ledger, K. (2018). Managing knowledge through social media: Modelling an entrepreneurial approach for Scottish SMEs and beyond. Baltic Journal of Management, 13(3), 303-328. doi: https://doi.org/10.1108/BJM-05-2017-0133

Denzin, N. K., \& Lincoln, Y. S. (2005). The SAGE Handbook of Qualitative Research. Thousand Oaks: Sage Publications.

Garretson, R. (2008). Future tense: The global CMO. The Economist, 1-33. Recuperado de http://graphics.eiu.com/upload/Google Text.pdf

Gutierrez-Leefmans, C., Nava-Rogel, R. M., \& Trujillo-Leon, M. A. (2016). Digital Marketing in an Emerging Country: Exploratory Study of the Marketing Mix of SMES with Trust Seal. Revista Brasileira de Marketing, 15(02), 207-219. doi: https://doi.org/10.5585/remark.v15i2.3117 
Villagómez Manrique, A. R. y Acosta Gonzaga, E. Uso de redes sociales digitales como estrategia de mercadotecnia en pymes pirotécnicas de Tultepec en el estado de México

Hanna, R., Rohm, A., \& Crittenden, V. L. (2011). We're all connected: The power of the social media ecosystem. Business Horizons, 54(3), 265-273. doi: https://doi.org/10.1016/j.bushor.2011.01.007

He, W. (2012). A review of social media security risks and mitigation techniques. Journal of Systems and Information Technology, 14(2), 171-180. doi: https://doi.org/10.1108/13287261211232180

Kaplan, A. M., \& Haenlein, M. (2010). Users of the world, unite! The challenges and opportunities of Social Media. Business Horizons, 53(1), 59-68.

Lashgari, M., Sutton-Brady, C., Solberg Søilen, K., \& Ulfvengren, P. (2018). Adoption strategies of social media in B2B firms: a multiple case study approach. Journal of Business \& Industrial Marketing, 33(5), 730-743. doi: https://doi.org/10.1108/JBIM-10-2016-0242

McCann, M., \& Barlow, A. (2015). Use and measurement of social media for SMEs. Journal of Small Business and Enterprise Development, 22(2), 273-287. doi: https://doi.org/10.1108/JSBED-08-2012-0096

Odoom, R., Anning-dorson, T., \& Acheampong, G. (2017). Antecedents of social media usage and performance benefits in small and medium sized enterprises (SMEs). Journal of Enterprise Information Management, 30(3), 383-399. https://doi.org/10.1108/JEIM-04-2016-0088

Ortega, S. (2018). La vida en Tultepec, donde la pirotecnia es una tradición a la que los pobladores no quieren renunciar. Sin Embargo. Recuperado de https://www.sinembargo.mx/20-08-2018/3459475

Pereault, W., \& McCarthy, J. (2002). Basic Marketing: A Global-Managerial Approach (14th ed.). New York: McGraw-Hill.

Rincón, S. (2016). Tultepec: el pueblo mexicano que vive de la pólvora. Univisión Noticias. Recuperado de https://www.univision.com/noticias/explosiones/tultepecel-pueblo-mexicano-que-vive-de-la-polvora

Ríos, L. (2018). Sin permiso de venta de pirotecnia, artesanos de Tultepec sufrirán pérdidas millonarias. El Heraldo. Recuperado de https://heraldodemexico.com.mx/estados/sin-permiso-de-venta-de-pirotecniaartesanos-de-tultepec-sufriran-perdidas-millonarias/

Rose, C. (2011). The Security Implications Of Ubiquitous Social Media. School of Management Publications College, 15(1), 35-40.

Scuotto, V., Del Giudice, M., Peruta, M. R. della, \& Tarba, S. (2017). The performance implications of leveraging internal innovation through social media 
Villagómez Manrique, A. R. y Acosta Gonzaga, E. Uso de redes sociales digitales como estrategia de mercadotecnia en pymes pirotécnicas de Tultepec en el estado de México

networks: An empirical verification of the smart fashion industry. Technological Forecasting and Social Change, (120), 184-194.

doi: https://doi.org/10.1016/j.techfore.2017.03.021

Solís, A. (2018). Tultepec y su explosiva industria de 300 millones de pesos. Forbes México. Retrieved from https://www.forbes.com.mx/tultepec-y-su-explosivaindustria-de-300-millones-de-pesos/

Tayouri, D. (2015). The Human Factor in the Social Media Security - Combining Education and Technology to Reduce Social Engineering Risks and Damages. Procedia Manufacturing, 3(Ahfe), 1096-1100. doi: https://doi.org/10.1016/j.promfg.2015.07.181

Villagómez-Manrique, A. R. (2019). Clasificación del sistema productivo de juguetería pirotécnica de Tultepec, Edo. de Méx. como un Distrito Industrial (Tesis de maestría). Instituto Politécnico Nacional. Recuperado de https://www.sepi.upiicsa.ipn.mx/oferta-educativa/maestria-enpymes/alumnos/graduados.html

Wamba, S. F., \& Carter, L. (2014). Social Media Tools Adoption and Use by SMES. Journal of Organizational and End User Computing, 26(2), 1-17. doi: https://doi.org/10.4018/joeuc.2014040101

Waters, R. D., Burnett, E., Lamm, A., \& Lucas, J. (2009). Engaging stakeholders through social networking: How nonprofit organizations are using Facebook. Public Relations Review, 35(2), 102-106.

doi: https://doi.org/10.1016/j.pubrev.2009.01.006

Yao, B., Shanoyan, A., Peterson, H. H., Boyer, C., \& Baker, L. (2018). The use of new-media marketing in the green industry: Analysis of social media use and impact on sales. Agribusiness, (35), 281- 297. https://doi.org/10.1002/agr.21581

Zhang, Z., \& Gupta, B. B. (2018). Social media security and trustworthiness: Overview and new direction. Future Generation Computer Systems, 86, 914-925. doi: https://doi.org/10.1016/j.future.2016.10.007

Zhou, L., Wu, W. P., \& Luo, X. (2007). Internationalization and the performance of born-global SMEs: The mediating role of social networks. Journal of International Business Studies, 38(4), 673-690.

doi: https://doi.org/10.1057/palgrave.jibs.8400282 
Villagómez Manrique, A. R. y Acosta Gonzaga, E. Uso de redes sociales digitales como

estrategia de mercadotecnia en pymes pirotécnicas de Tultepec en el estado de México

\section{AUTORES:}

\section{Alan Roberto Villagómez Manrique}

Licenciado en Física. Estudiante del programa de la Maestría en Ciencias en Estudios Interdisciplinarios en pequeñas y medianas empresas, del Instituto Politécnico Nacional. Programa incluido en el PNPC de CONACYT.

phys.villa@outlook.com

Orcid ID: https://orcid.org/0000-0001-8294-5646

Google Scholar: https://scholar.google.com.mx/citations?user=IUTF -

oAAAAJ\&hl=es

\section{Elizabeth Acosta Gonzaga}

Doctora en Investigación Educativa. Docente e Investigadora de la Maestría en Ciencias en Estudios Interdisciplinarios en pequeñas y medianas empresas del Instituto Politécnico Nacional. Programa incluido en el PNPC de CONACYT. eacostag@ipn.mx

Orcid ID: https://orcid.org/0000-0001-5413-1063

Google Scholar:

https://scholar.google.es/citations?user=qPGIoZ8AAAAJ\&hl=es\&oi=ao 
Villagómez Manrique, A. R. y Acosta Gonzaga, E. Uso de redes sociales digitales como

estrategia de mercadotecnia en pymes pirotécnicas de Tultepec en el estado de México

\section{Anexo}

Cuestionario utilizado para la recolección de datos

Los objetivos del presente cuestionario son conocer el nivel de adopción de redes sociales, su uso y los beneficios percibidos por parte de los distribuidores de pirotecnia de Tultepec, Estado de México.

Confidencialidad: se garantiza que por ningún motivo se revelará información específica de esta encuesta, ni información que pueda revelar la identidad u opinión del encuestado. El tratamiento de los datos será estadístico y con propósitos académicos.

\section{Cuestionario}

No. de

Fecha:

Años en el negocio:

encuesta:

Edad:

1. ¿Cuál es su función principal dentro de la actividad pirotécnica?

Fabricante Mayorista Minorista Agente Otro:

2. En total, ¿cuántas personas trabajan en su taller/empresa/local?
De 0 a 10
De 11 a 50
De 51 a 250

Más de 250

3. ¿Su negocio se encuentra registrado en alguna red social digital?
SI
NO. (Pasar a la pregunta 15)

4. ¿En qué red social se encuentra registrado? (Puede elegir más de una opción) Facebook

Instagram

Twitter

YouTube

Otro:

5. ¿Cuánto tiempo lleva utilizando redes sociales para su negocio?

Menos de De 1 a 3 años De 3 a 5 años De 5 a 10 año Más de 10 años 1 año

6. ¿Con qué frecuencia utiliza las redes sociales de su negocio?

7. ¿Contrató personal especializado para la creación y administración de su red social digital?

SI.

NO.

8. ¿Quién es el encargado de administrar sus redes sociales?

Dueño $\begin{gathered}\text { Trabajador } \\ \text { especializado }\end{gathered}$ Trabajador NO Agencia Familiar Otro:
especializado 
Villagómez Manrique, A. R. y Acosta Gonzaga, E. Uso de redes sociales digitales como estrategia de mercadotecnia en pymes pirotécnicas de Tultepec en el estado de México

9. En la siguiente tabla, marque con una 'x' la opción que corresponda con el uso que da a sus redes sociales digitales.

\begin{tabular}{|c|c|c|c|}
\hline Uso & $\begin{array}{c}\text { Nunca/poco } \\
\text { frecuente }\end{array}$ & Frecuente & $\begin{array}{c}\text { Siempre/muy } \\
\text { frecuente }\end{array}$ \\
\hline Dar información sobre el uso de los productos & & & \\
\hline Dar Información de las características de los & & & \\
productos & & & \\
\hline Dar descuentos en los productos & & & \\
\hline Dar información de los precios de los productos & & & \\
\hline Vender a intermediarios & & & \\
\hline Vender a clientes finales/consumidores & & & \\
\hline Dar demostraciones virtuales de los productos & & & \\
\hline Mejorar sus relaciones con sus clientes & & & \\
\hline Anunciar eventos & & & \\
\hline Dar asistencia en línea & & & \\
\hline Recibir opiniones sobre los productos/servicios & & & \\
\hline Otro: & & & \\
\hline
\end{tabular}

10. ¿Qué beneficios ha obtenido su negocio gracias al uso de redes sociales digitales? Incremento Mayor popularidad Mejor relación con Otros: de ventas de su marca los clientes

11. ¿Ha conseguido nuevos clientes a través de redes sociales digitales?

Si No. (Pasar a la pregunta 13)

12. ¿De qué región son los clientes conseguidos por medio de redes sociales digitales? Mismo municipio Otro municipio Otro estado Otro país

13. ¿Recomendaría el uso de redes sociales digitales a otras empresas?

$\mathrm{Si}$

No.

14. ¿Por qué?

15. ¿Por qué no utiliza redes sociales digitales para su negocio? 